\title{
Lapurdum
}

Euskal ikerketen aldizkaria | Revue d'études basques |

Revista de estudios vascos | Basque studies review

$8 \mid 2003$

Numéro VIII

\section{Précisions sur la Guerra grande et ses répercussions sur les immigrés originaires de France et du Pays basque}

\section{Claude Méhats}

\section{OpenEdition}

Journals

Édition électronique

URL : http://journals.openedition.org/lapurdum/1154

DOI : 10.4000/lapurdum. 1154

ISSN : 1965-0655

Éditeur

IKER

Édition imprimée

Date de publication : 1 novembre 2003

Pagination : 349-358

ISBN : 9782867813436

ISSN : $1273-3830$

Référence électronique

Claude Méhats, «Précisions sur la Guerra grande et ses répercussions sur les immigrés originaires de France et du Pays basque », Lapurdum [En ligne], 8 | 2003, mis en ligne le 01 mai 2009, consulté le 04 mai 2019. URL : http://journals.openedition.org/lapurdum/1154; DOI : 10.4000/lapurdum.1154 


\section{Claude Méhats}

Doctorant en histoire

\section{Précisions sur la Guerra grande et ses répercussions sur les immigrés originaires de France et du Pays basque}

Après l'article paru dans le numéro précédent de Lapurdum (Méhats, 2002), il nous a semblé utile d'apporter quelques précisions pouvant aider à une meilleure connaissance de la guerre civile uruguayenne (1842-1851) communément dénommée Guerra grande. Un retour sur les articles en langue basque parus en 1843 dans le Patriote Français de Montevideo' et sur leur impact précèdera des strophes composées en basque, dont l'origine nous est inconnue, mais que nous avons pu mettre en rapport avec un fait historique avéré.

Lorsque la situation du général José Fructuoso Rivera devint critique en conséquence de la défaite d'Arroyo Grande, on commença en toute hâte la fortification de Montevideo car aucune troupe ne semblait en mesure de stopper l'avancée des troupes de Manuel Cerefino Oribe. A Montevideo, le relais de la politique de Guizot était assuré par le consul du roi, Théodore Pichon. Alors que le conflit avait lieu en province, le Patriote Français n'hésita pas à rappeler que son devoir était avant tout de protéger ses administrés et qu'avec l'approche de la menace, Th. Pichon disposait là d'une bonne occasion de se signaler. Effrayé à l'idée que ses compatriotes puissent être sacrifiés « sous le vain prétexte de la médiation", le rédacteur du journal leur demandait de porter sur eux de manière distinctive les couleurs françaises et leurs papiers ${ }^{2}$. Une semaine plus tard, le consul organisa une réunion où il lut une dépêche ministérielle indiquant «qu'il est inutile que la population française prenne les armes et que si une intervention paraît utile au gouvernement, les forces

\footnotetext{
Nous tenons à remercier Alberto Irigoyen Artetxe qui d'une part a révélé l'existence de ces articles, mais qui a aussi été un soutien précieux dans les recherches menées en Uruguay, tant par ses indications que par son soutien matériel.
}

2 Le Patriote Français, dimanche 5 février 1843, n 3, p. I 
nécessaires seront dirigées sur le Rio de la Plata. » Des signaux furent émis entre le consulat et les bâtiments de guerre qui restaient vigilants, et il était recommandé de signaler les maisons françaises par des drapeaux tricolores ${ }^{3}$.

Le lendemain, toujours au consulat, une réunion fut organisée sous la houlette de Théodore Pichon. Il convint en accord avec les Français de faire le nécessaire pour que leurs droits fussent respectés. Ceci se traduisait par une « neutralité armée, respectueuse envers le pays et ses autorités et profitable à la sûreté générale 4 . " Les Français furent avisés par voie de presse. Des réunions eurent lieu dans tous les quartiers où ils étaient nombreux et on leur lut le rapport de la commission (parmi les points de rencontre: "Jeu de paume, Cazenave; Maison Recaite; Jeu de paume, Capandeguy 5 ") Au sein des membres de la commission présidée par le consul apparurent les personnages les plus motivés dont Jean-François Thiebaut, Martin Cazenave et Salvate Etchegoyen ${ }^{6}$. Les Français étaient donc mobilisés dans une «neutralité armée». Des articles furent publiés en langue basque pour tenter de rallier les immigrés basques à la défense de Montevideo (Méhats, 2002).

Le dimanche 19 février, le journal opposa «à quelques assertions mensongères " qui voulaient faire croire que le consul restait étranger à l'armement des ressortissants français, le premier appel que ce dernier avait lancé? appel dans lequel il exhortait les Français à s'armer. Dans les jours suivants, Le Patriote Français reconnut pourtant que le consul avait changé de position, reculé dans son engagement, et s'en offusqua. Théodore Pichon tenta de freiner les initiatives de ses administrés en leur rappelant l'article 21 du code civil qui stipulait que « le Français qui, sans autorisation du roi, prendrait du service militaire chez l'étranger, ou s'affilierait à une corporation militaire étrangère, perdra la qualité de Français ». Il se dégagea de ses responsabilités vis-à-vis de tous ceux qui avaient l'intention de prendre part aux combats en faveur de l'Uruguay. La barrière législative dressée par le représentant du gouvernement français n'était pas un obstacle aux yeux de tout le monde, puisque dans le même numéro, la lettre d'un lecteur légitimait la prise

\footnotetext{
${ }^{3}$ Le Patriote Français, samedi 11 février 1843, n ${ }^{\circ}$ 8, p. 2

${ }^{4}$ Le Patriote Français, dimanche 12 février 1843 , n 9, p. 3

5 Le Patriote Français, lundi 13 et mardi 14 février $1843, n^{\circ} 10$

${ }^{6}$ Le Patriote Français, mercredi 15 février 1843, n 11, pp. 1-2

${ }^{7}$ Le Patriote Français, dimanche 19 février 1843, n 15, p. 1
} 
d'armes et la désobéissance au consul comme un héritage issu de la révolution française. En accomplissant ce qui lui paraissait juste et en désobéissant à de mauvaises décisions, il estimait être de la sorte proche des révolutionnaires qui prirent la Bastille en $1793^{8}$ (sic!).

Dans le quatrième article intitulé Adaisquide eta herritar maitiac, les défenseurs de Montevideo aigris par la volte-face du consul de France Théodore Pichon signalèrent qu'ils avaient néanmoins pu faire entendre leur voix à un noble de haut rang, le prince de Joinville. François Ferdinand Philippe d'Orléans ${ }^{9}$, prince de Joinville était le troisième fils du roi LouisPhilippe. Capitaine de vaisseau en 1839, c'est lui qui fut chargé en 1840 de ramener de Sainte-Hélène les restes de Napoléon Ier. Son opposition à Guizot lui valut une grande popularité et c'est à ce titre que les Français assiégés dans Montevideo et opposés à la politique de leur gouvernement en firent leur interlocuteur légitime.

Dans un avis adressé aux Français, inquiet par la persistance de rumeurs annonçant la création d'un bataillon par ses compatriotes, Théodore Pichon rappela les articles 84 et 85 du code pénal qui prohibaient ces agissements et exposaient à des peines ceux qui les enfreignaient. Avec des préoccupations qui au premier regard peuvent sembler plus pratiques, un lecteur interpella le rédacteur du Patriote en lui demandant ce qu'il adviendrait des " 7 à 800 Basques, nouvellement arrivés », qui ne pouvaient trouver de travail et ne devaient donc assurer leur salut qu'en contractant un engagement dans l'armée ${ }^{10}$.

Après que le gouvernement oriental eut appelé les étrangers à venir se battre dans ses rangs, le consul de France rétablit la communication. Il rappela une nouvelle fois les articles 84 et 85 du code civil:

«Article 84 - Quiconque aura, par des actions hostiles non approuvées par le gouvernement, exposé l'état à une déclaration de guerre, sera puni du bannissement, et, si la guerre s'en est suivie, de la déportation ».

Article 85 - Quiconque aura, par des actes non approuvés par le gouvernement, exposé des Français à éprouver des représailles, sera puni du bannissement »

\footnotetext{
${ }^{8}$ Le Patriote Français, mercredi 22 février $1843, \mathrm{n}^{\circ} 17$, pp. 2-3

9 Né à Neuilly le 14 août 1818 et décédé à Paris le 16 juillet 1900 .

${ }^{10}$ Le Patriote Français, dimanche 5 mars 1843, n 26, pp. 1-2
} 
Le rédacteur du Patriote rétorqua immédiatement, considérant que les Français étaient en position d'agressés et que le mot de représailles était inexact car Rosas comme Oribe pratiquaient les meurtres et les pillages ${ }^{11}$.

Le divorce entre le consul et l'opinion publique fut consommé le samedi 15 avril 1843, alors que sous une pluie battante, une centaine de Basques se pressaient devant le consulat en espérant trouver un abri. La porte fut entrouverte pour uniquement laisser entrer un chien. Exaspéré par le changement d'attitude du consul, Le Patriote Français entama à son encontre une campagne systématique de dénigrement ${ }^{12}$.

Tous les articles qui suivirent, concernant Théodore Pichon, n'eurent: qu'un seul objectif: le présenter comme un traître qui permit à une trentaine de Basques-Français de quitter l'Uruguay sans papiers ${ }^{13}$. Il donna son aide à des Basques-Espagnols, les considérant comme des Basques-Français ${ }^{14}$, mais refusa de l'apporter aux Basques de France. Il ordonna aux Français de quitter les armes ${ }^{15}$. C'est au début de l'année 1844 que parut le dernier article qui l'attaquait ${ }^{16}$. Le consul demanda que la Légion Française fût licenciée pour respecter le principe de neutralité, puis il embarqua à bord d'un navire de guerre. Pour les rédacteurs du Patriote Français, il n'était plus considéré que comme un félon.

En mai 1843, une rumeur circula quant au départ de Théodore Pichon et à son remplacement par $\mathrm{M}$. de Lesseps ${ }^{17}$. L'enthousiasme gagna rapidement les rangs de ses adversaires qui imaginaient une future intervention de la France dans la Guerra grande. Dans l'euphorie, M. de Saint Chartrier composa deux chants pour fêter le départ hypothétique du consul: Chant $d u$ départ ${ }^{18}$ et $M$. Pichon s'en val9. Le refrain de la première cuvre ne laisse

${ }^{11}$ Le Patriote Français, dimanche 9 avril 1843, n ${ }^{\circ} 55$, pp. 1-2

12 Le Patrioiu Français, lundi 17 et mardi 18 avril $1843, \pi^{\circ} 60$, p. 1

${ }^{13}$ Le Patriote Français, dimanche 23 avril 1843, n 65, p. 3 ; Le Patriote Français, lundi et mardi 25 avril $1843, n^{\circ} 65^{1 / 2}$, p. 1 ; Le Patriote Français, lundi et mardi 13 juin 1843, $n^{\circ} 105$, pp. 1-2; Le Patriote Français, mercredi 14 juin 1843, n 106 , pp. 1-2

${ }_{14}$ Le Patriote Français, mercredi 31 mai 1843, n 94, p. 1; Le Patriote Français, lundi et mardi 25 juillet $1843, \mathrm{n}^{\circ} 139$, pp. 2-3; Le Putriote Français, samedi 23 septembre 1843, n 190, p. 2; Le Patriote Français, mercredi 11 octobre $1843, n^{\circ} 205$, p. 2

15 Le Patriote Français, vendredi 15 septembre 1843, n 183, pp. 1-2

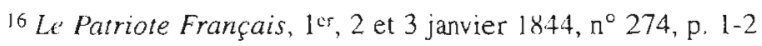

${ }^{17}$ Le Patriote Françuis. mercredi 24 mai $1843, n^{\circ} 89$, p. 1

${ }^{18}$ Le Patriote Français, vendredi 26 et samedi 27 mai $1843, \pi^{\circ} 91$. p. 2

19 Le Patriote Français, lundi 29 et mardi 30 mai $1843, n^{\circ} 93$, p. 2 
place à aucune ambiguité sur la considération que le compositeur portait au diplomate:

« Bon voyage

Monsieur de Pichon

A Paris débarquez sans naufrage

Bon voyage

Monsieur de Pichon

Et qu'on n'entende ici plus votre nom. »

Lors de la prise d'armes des Volontaires français, l'enthousiasme de leurs compatriotes pyrénéens resta très mesuré, et il fallut les informer en basque. La parution des articles dans le Patriote Français ne fit pas évoluer la situation de manière considérable, et il fallut l'intervention orale d'un négociant et la proposition de créer un bataillon particulier pour que les Basques s'investissent en masse dans la défense de Montevideo ${ }^{20}$. Quant à la tenue, le bataillon des Chasseurs basques reçut un uniforme spécial, compo-

\footnotetext{
2 (1) "Cependant, on remarquait que la population basquaise et béarnaise n'apportait pas le même empressement à se faire inscrire que les résidents venus des autres contrées de la France. Les premiers surtout, soit par un sentiment de nationalité qu'on ne retrouve pas chez les autres habitants de nos provinces, soit que l'idiome de leur pays natal, qu'ils estiment au-dessus de tout autre langage. les génât ou leur fît appréhender de se mêler à ceux de leurs conipatriotés qui ne les comprenaient pas, ils s'abstenaient en grande partie et figuraient peu, comparativement à leur nombre, sur les registres d'enrôlement. Ce serait, du reste, insulter à leur valeur, dont ils ont donné tant de preuves, que de supposer que s'ils se hâtaient pas, c'est qu'ils ne partageaient pas les sentiments de leurs conationaux.
}

Le colonel Thiebaut donna un ordre du jour afin de stimuler l'ardeur attardée des Basques. Cet ordre, bien que traduit en langue basquaise, ne produisit qu'une partie de l'effet qu'on en attendait. Un honorable négociant, comprenant parfaitement de quelle importance serait l'admission en grand nombre de ces hommes laborieux autant qu'alertes et courageux, se chargea de répondre à l'appel fait à la population basquaise, et le projet d'armement provoqué par l'ordre du jour, ce négociant, qui était Basque lui-même, répondit tout en entrant dans les vues du projet:

...«Mais pour cela, dit-il, il faut que les Basques forment un corps pariculier qui prendra le nom de Chasseurs Basques, dont tous les chefs et les soldats seront Basques; alors, la difficulté qui les retient étant levée, on les verra accourir de toutes parts pour offrir l'appui de leurs bras et leur courage. Les descendants des Cantabres, les compagnons d'armes de Latour d'Auvergne, les compatriotes du brave général Harispe, prouveront qu'ils sont aussi vaillants sur les rives de la Plata, qu'ils l'ont été sur les bords de la Bidassoa. "(Patriote Français du 13 avril 1843).

Celui qui écrivait ces lignes connaissait bien ses compatriotes; car à peine cette idée étaitelle êmise, et les listes d'enrôlements ouvertes, qu'elles se couvrirent de noms basques, et le troisième bataillon fut formé.

Un quatrième bataillon, et même un cinquième, furent spontanément organisés. Ce dernier, presqu'exclusivement composé de Basques, se fondit dans le troisième, qui devint à son tour, deux ans plus tard, le REGIMENT DES CHASSEURS BASQUES. " (Lefère, 1852, 51) 
sé d'une veste ronde avec un plastron, d'un pantalon bleu et d'un béret rouge (Lefèvre, 1852, 60).

Plusieurs Basques, menant déjà les compagnies, briguaient le commandement du bataillon des Chasseurs basques. Etchardt, Brie, Oyenard et Labastie se faisaient concurrence, les plus avantagés étant les trois premiers grâce au rôle actif qu'ils avaient joué dans la formation du bataillon. Etchardt l'emporta, mais il ne resta pas longtemps en poste, « une intrigue dans laquelle il se trouva impliqué le renversa, et le dégoût s'emparant de son âme, il ne traîna plus qu'une misérable existence que la mort vint abréger peu de temps après ». (Lefèvre, 1852, 53). Oyenard lui succéda, mais la dissolution du cinquième bataillon dont il était le commandant l'entraina vers l'Etat-Major, en qualité d'organisateur et d'administrateur jusqu'à ce qu'il quittât la Légion. C'est ainsi que le commandement échut à Jean-Baptiste Brie ${ }^{21}$.

Après avoir éclairci quelques points relatifs à la parution des articles en langue basque dans le Patriote Français de Montevideo de 1843 ainsi que la création du régiment des Chasseurs basques, nous allons maintenant nous intéresser à un épisode méconnu de l'histoire sud-américaine en prenant comme source une chanson de bertsulari. La chanson Mercedes-etako berriak 1845 (Les nouvelles de Mercedes 1845) est une œuvre non attribuée. Elle contient huit strophes en zortziko ttipi. L'action se situe en Uruguay, dans la région entre Durazno et Mercedes, le jeudi 11 septembre 1845. Un groupe de personnes se sentant probablement en danger dans la première ville, prit la fuite ( Sortant de Durazno au crépuscule 22 ) mais fut rattrapé par des tueurs à gage, volé une première fois, puis dépouillé durant une seconde intervention «à la vallée de Bekelu ${ }^{23}$ » par une troupe armée et bien organisée («Des sentinelles à cheval devant et derrière $\left.{ }^{24} »\right)$.

\footnotetext{
${ }^{21}$ Jean-Baptiste Brie s'installa comme médecin à Montevideo en 1838. Il semble qu'il naquit en 1797 à Saint-Jean-Pied-de-Port et qu'il ait fait ses études de médecine à Montpellier. En 1839, il apparut pour la première fois dans la liste des médecins en exercice à Montevideo. Durant la Guerra Grande, il fut colonel dans l'armée uruguayenne et son frère Dominique capitaine des chasseurs basques. Après le conflit, Jean-Baptiste Brie se retira à Peñarol où il continua à pratiquer la médecine. Fidèle à ses convictions, il mourut assassiné en 1858, dans un des épisodes révolutionnaires qui caractérisa le XIX ${ }^{e}$ siècle uruguayen. (Fernandez Saldana, 1945, 235-236)
}

22 Durasno-tik athera ilhunbarrian (strophe 2, vers 1)

${ }^{23}$ Bekeluko errekan (strophe 6, vers 1)

${ }^{24}$ Aitzin gibeletarik sentinelak zaldiz (strophe 5, vers 3) 
Cette chanson fait référence à un véritable événement historique, celui des otages de Durazno. Douze jours avant le blocus franco-anglais du 24 septembre 1845, le général Manuel Oribe donna l'ordre de confiner tous les Français et les Anglais des départements du Littoral: Colonia, Soriano, Paysandu... dans la ville de Durazno à l'intérieur du pays. C'est ainsi que des Européens résidant à Mercedes furent arrêtés en pleine nuit et conduits (sans avoir eu le temps de se vêtir) au centre de la ville. Le lendemain, quelquesuns purent rentrer chez eux pour aller prendre leurs effets personnels. L'exode vers Durazno commença et au bout de trois lieues, les troupes entreprirent le dépouillement de leurs prisonniers. Au cours du déplacement qui dura dix jours, les Anglais et Français devaient passer les rivières à pied et "M. François, un basque français » fut égorgé dans les environs du ruisseau de Coquimbo. De la soixantaine d'étrangers résidant à San Salvador et conduits par un détachement du lieutenant Hilario Gonzalez, vingt-trois furent séparés de la colonne. Huit d'entre eux furent confiés au capitaine Ludueña qui les fit poignarder et égorger. Ils furent jetés dans la lagune avec des pierres attachées au cou. Les quinze autres arrivèrent liés à Durazno, attachés par le cou. Sur les 240 étrangers qui passèrent onze mois dans le camp de de Durazno, 229 étaient originaires de France et 11 d'Angleterre. Sur les dix-huit d'entre eux qui moururent durant leur captivité treize étaient originaires de villages basques des Basses-Pyrénées (Bergès, 1937, 4).

Pour conclure, nous pouvons signaler que beaucoup de recherches restent à effectuer sur le rôle et l'importance de la présence basque dans la guerre civile uruguayenne. Dans ce texte comme dans le précédent, ce sont les Basques originaires des provinces de France qui ont été privilégiés mais il faut garder à l'esprit que ceux originaires des quatre provinces d'Espagne se battaient pour le compte d'Oribe. Les archives du bataillon des Chasseurs basques n'ont pas encore été découvertes et il faut compter sur l'apparition de tels documents pour parfaire nos connaissances qui restent encore floues en particulier quant aux causes de la séparation et de la brouille survenue en 1845 entre les Chasseurs basques et la Légion française. L'épisode de Durazno, relaté par la chanson Mercedes-etako berriak 1845, fait référence à l'histoire du premier camp de concentration d'Amérique du sud. 


\section{Mercedes-etako berriak 1845}

Mila zortzi ehun eta berrogoi eta bortza, Burularen hamekan zen orzegun goiza; Jeinkoak entzun balu guk egin othoitza, Ez ginduen izanen dugun zori gaitza.

Durasno-tik athera ilhunabarrian, Egia erraiteko kasik ilhunian, Oren on bat bezala emanik bidian, Gaba kanpuan pasatu etzanik urian.

Etzen uste gabe jin biharamun goiza, Lau hatzetan ikharan ezin kenduz hotza, Bixtan da gisa hartan ez ginela ontsa, Orduan juan zioten Batiztari sosa.

Ontzako urhiak zituen berrogoi eta zazpi, Athorraren barnian zinturan ez gaizki, Abisatuak ziren duda gabe naski, Heien denen kentzia oraino etzen aski.

Durasno-ko errekan formaturik berriz, Orduan etzagoen jendia han irriz, Aitzin gibeletarik sentinelak zaldiz, Bizia gal beldurrez gu ikharan aldiz.

Bekeluko errekan zen berriz apela, Ordukotz bazakiten diruk etzela, Pontxuak-emaiteko kuidado bertzela, Aitzindarietarik ordena zutela.

Xaleko eta gerriko bai eta ere bota, Athorra kalzonzilo horiek ez khonda, Aitzindariek hala ordena eman eta, Ohointza utzi zuten guziak kendu eta.

Horra zer berri dugun, oi! Mercedes-etan, Nik aditzia dudanaz orobat bertzetan, Eman izan gaituzte ondikotz penetan, Malerus izaiteko gu Ameriketan! 


\section{Les nouvelles de Mercedes 1845}

En mille huit cent quarante cinq,

Le onze septembre au jeudi matin;

Si Dieu avait entendu la prière que nous avons faite,

Nous n'aurions pas eu cette mésaventure.

Sortant de Durasno au crépuscule,

A dire vrai presqu'à la nuit,

Ayant mis près d'une heure sur le chemin,

On a passé la nuit couché dans l'eau.

Le lendemain matin n'était pas venu par surprise,

Tremblant des quatre membres n'arrivant pas à chasser le froid,

Il est évident que de cette façon nous n'étions pas bien,

C'est à ce moment là qu'ils emportèrent l'argent à Baptiste.

Il avait quarante-sept onces d'or,

Elles n'étaient pas mal dans la ceinture à l'intérieur de la chemise,

Ils étaient avertis sans aucun doute je pense,

Çà ne suffisait pas encore de toutes les enlever.

Dans la rivière de Durasno glacés de nouveau,

Il n'était pas alors là-bas question de rire,

Des sentinelles à cheval devant et derrière,

Tandis que nous tremblions de perdre la vie.

Nouvel appel dans la vallée de Bekelu,

Pour lors ils savaient qu'il n'y avait pas d'argent,

Il fallait donner nos ponchos sinon gare!

Que les chefs en avaient donné l'ordre.

Veston et ceinturon et aussi les bottes,

Chemise et caleçon ça ne compte pas,

Les chefs ont donné cet ordre et,

Ils cessèrent de voler après nous avoir tout enlevé.

Voilà quelles sont les nouvelles, hélas! à Mercedes,

De même ailleurs d'après ce que j'ai entendu,

Ils nous ont mis hélas dans la peine,

Pour que nous soyons malheureux en Amérique! 


\section{Bibliographie}

BERGES, Pedro, 1937 : "Le martyrologue Basque-français en Uruguay. Les assassinats du corralito, du Dr Brie de Laustan et du président Idiarte Borda"; Le courrier de la Plata (numéro spécial du 14 juillet), 15 pp.

FERNANDEZ SALDANA, J. M., 1945 : Diccionario uruguayo de biografias (1810-1840) Montevideo.

LEFEVRE, J., 1852 : La légion française, première année du siège de Montevideo. Extrait des souvenirs d'un volontaire, Montevideo: Imprimerie du « Patriote Français", 126 pp.

MEHATS, Claude, 2002 : "Articles en basque parus en 1843 dans le Patriote Français de Montevideo", Lapurdum 7, 247-257.

POUCEL, Benjamin, 1854: Cartas criticas. Estudio historico de las razas latinas y anglo-sajona. Necesidad de una educacion nacional en la America del sud, Montevideo: Imprenta del rio de la Plata, 102 pp.

POUCEL, Benjamin, 1864 : Les otages de Durazno. Souvenirs du Rio de la Plata pendant l'intervention Anglo-Française de 1845 à 1851, Paris, Achille Faure libraire éditeur, 86 pp. 